\title{
A Statistical Description of CdSe/CdS Dot-in-Rod Heterostructures Scanning Transmission Electron Microscopy
}

\author{
Benjamin T. Diroll, Natalie Gogotsi, and Christopher B. Murray*
}

*cbmurray@sas.upenn.edu

Materials. Trioctylphosphine (90\%, Aldrich), trioctylphosphine oxide (99\%, Strem), tetradecylphosphonic acid (PCI), octadecylphosphonic acid (PCI), hexylphosphonic acid (PCI), selenium powder (Aldrich, 99.99\%), sulfur (99.99\%, Aldrich), and cadmium oxide (99.99\%, Strem) were purchased from commercial sources as specified and used as received without further purification. All solvents were purchased as ACS grade or higher. TEM grids were carbon-coated copper purchased from Electron Microscopy Sciences.

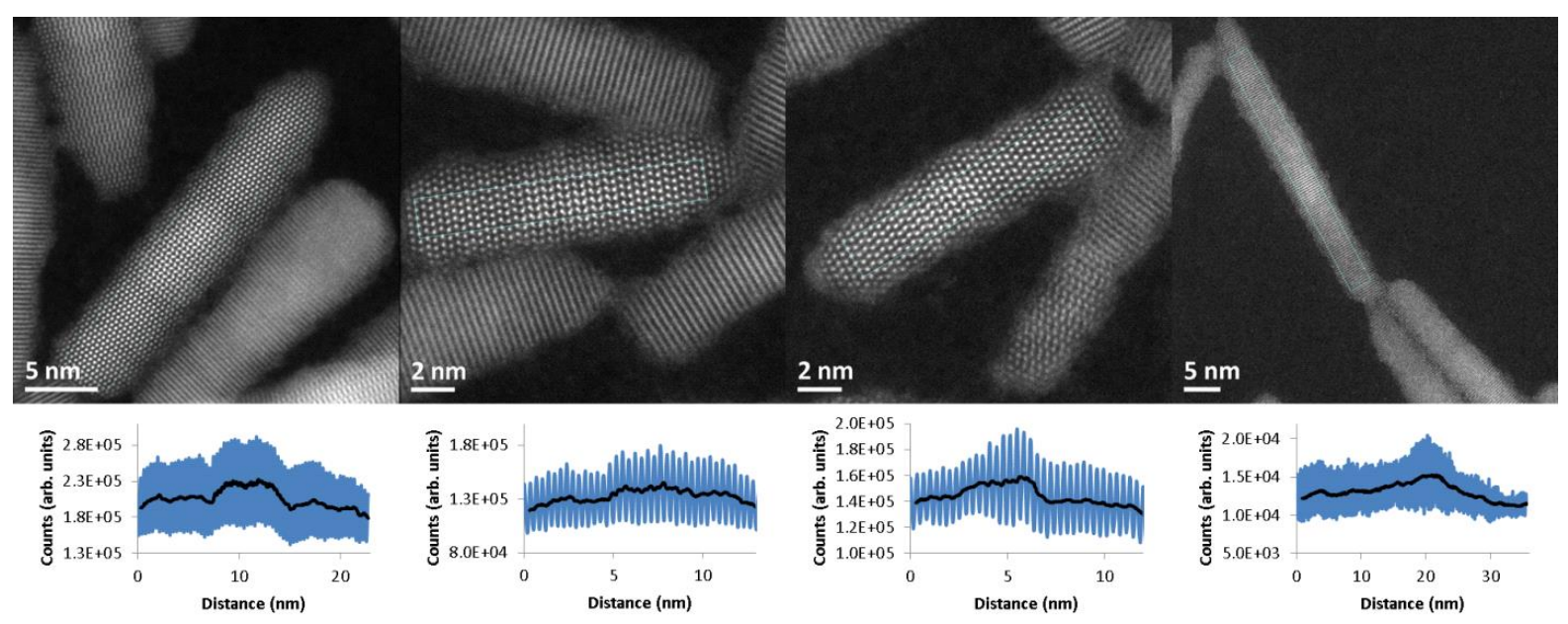

Figure S1. ADF-STEM images of the "large", "medium", "small", and "rod" core samples. Below each image in blue is an intensity profile from each rod at the center of the image, the black line is a moving average that suppresses intensity oscillations highlighting the position of CdSe core and showing the relative change in contrast with the core size. 


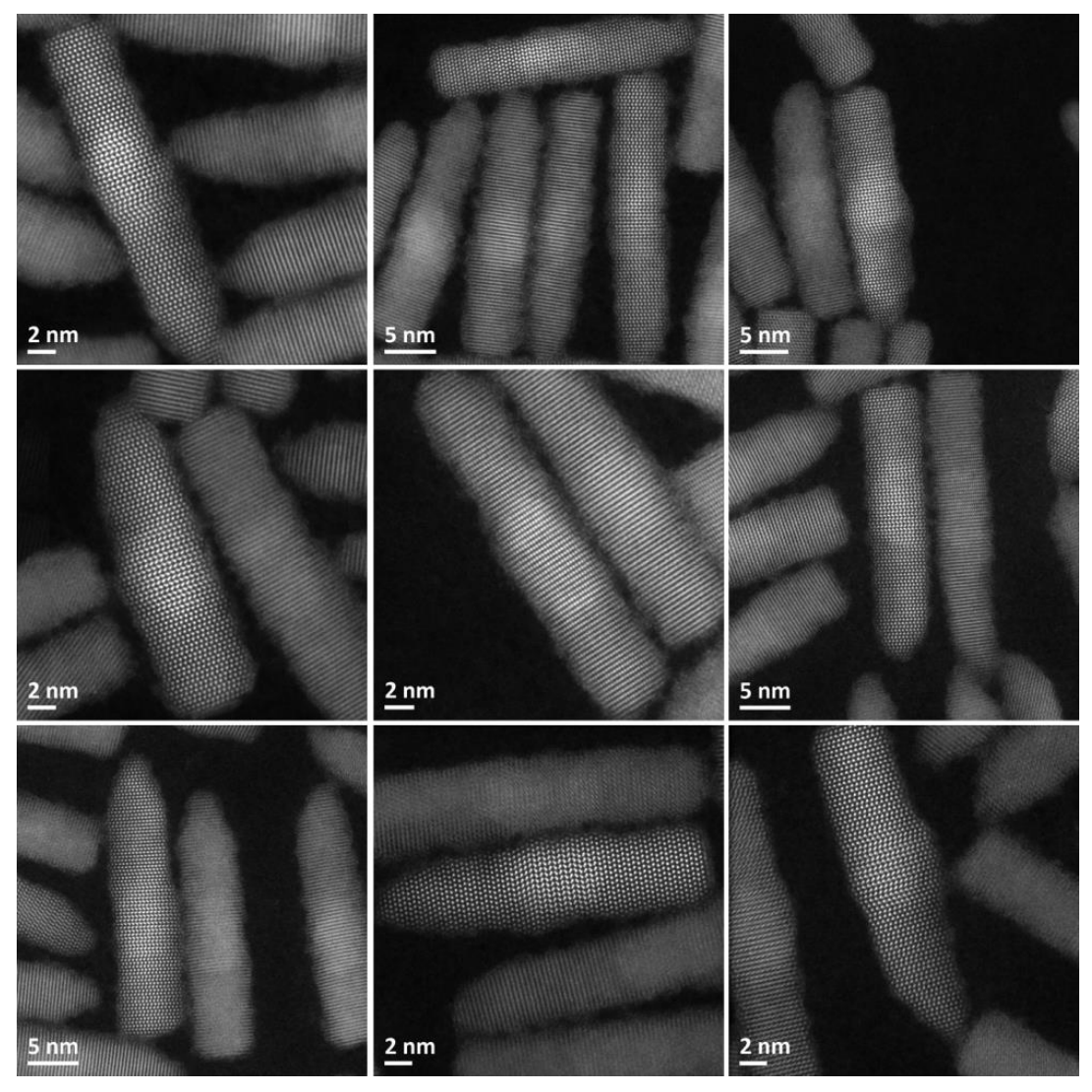

Figure S2. Panel of several ADF-STEM images of "large" core dot-in-rod heterostructures. 


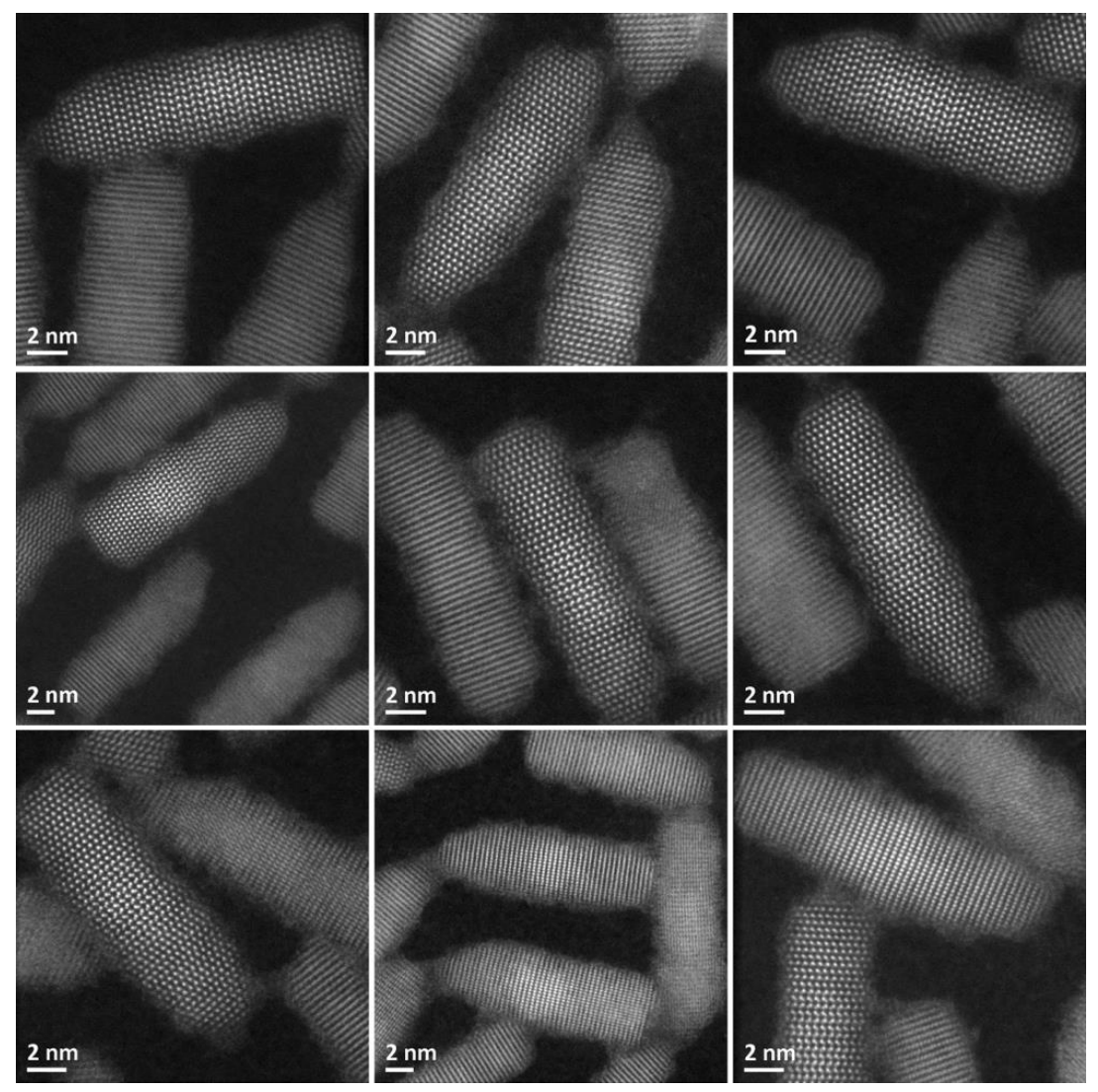

Figure S3. Panel of several ADF-STEM images of "medium” core dot-in-rod heterostructures. 


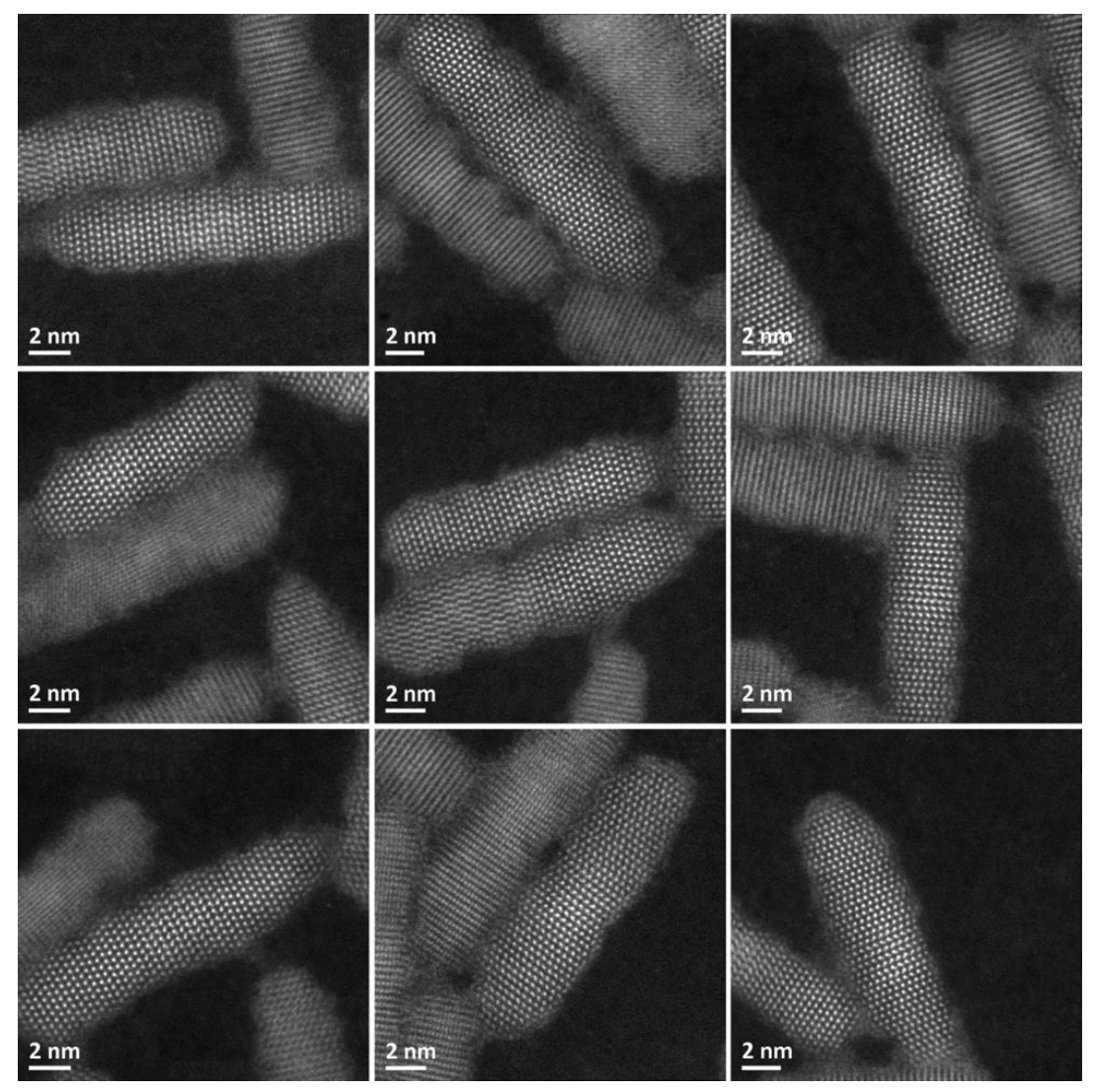

Figure S4. Panel of several ADF-STEM images of "small" core dot-in-rod heterostructures. 

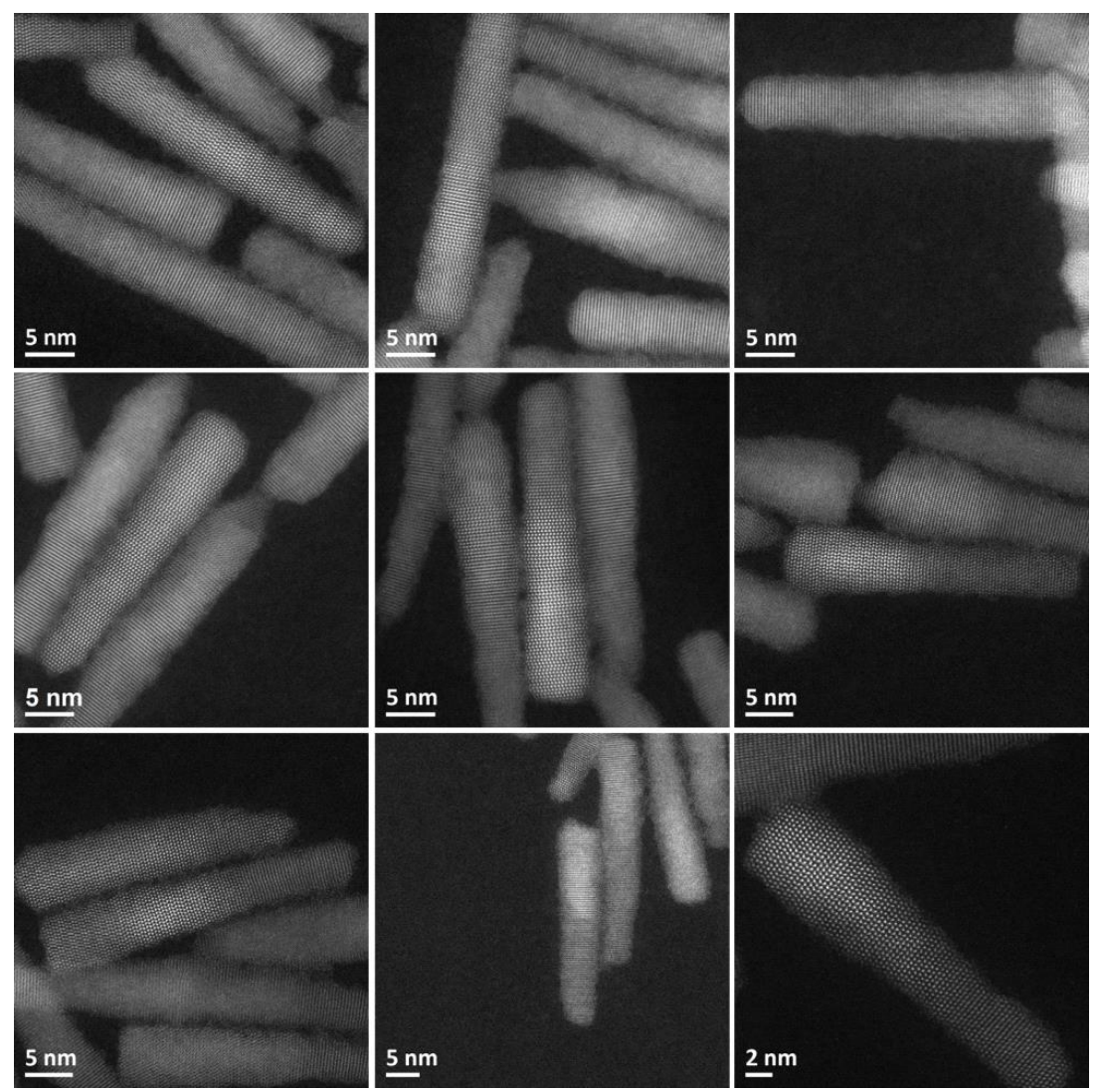

Figure S5. Panel of several ADF-STEM images of rod core rod-in-rod heterostructures.

Thermal History Experiments. The rod morphology was found to be strongly-dependent on the thermal history of reactions. To test the influence of stable temperature on the resulting morphology, we performed three experiments: (1) allow the reaction to cool to $<200{ }^{\circ} \mathrm{C}$ after seed injection, then restored to $350{ }^{\circ} \mathrm{C}$ for 3 minutes of reaction; (2) the reaction pot was allowed to recover normally to the set point temperature after injection, but after $90 \mathrm{~s}$, the temperature was dropped $<200{ }^{\circ} \mathrm{C}$ and then returned to $350{ }^{\circ} \mathrm{C}$ for $90 \mathrm{~s}$ further growth; and (3) a reaction run as a control in which the precursor and seed concentrations were the same as (1) and (2), but the reaction was allowed to run for 3 minutes after injection, returning to the set point temperature and not purposefully disrupted. Despite the difficulty of finely controlling temperature during a synthesis based on hot injection, the results of the thermal history experiments yield obvious conclusions. First, the length monodispersity of the samples is substantially different although the average lengths are similar. For reactions (1) and (2), the values of the NR length were $(44.3 \pm 11.3) \mathrm{nm}(\sigma=25 \%)$ and $(52.6 \pm 12.0) \mathrm{nm}(\sigma=23$ $\%)$, respectively. For the control reaction (3), the length was $(52.0 \pm 4.9) \mathrm{nm}(\sigma=9.4 \%)$. Second, as demonstrated in Figure S6, significant drops in temperature during the reaction lead to a large fraction of bulbous NRs. Analysis of $>200$ NRs for each sample found that reaction (1) contained $36 \%$ bulbous NRs, (2) contained $28 \%$ bulbous NRs, and the control (3) contained $4 \%$ bulbous NRs. These experiments indicate the critical nature of stable reaction temperatures in the formation of high-quality particles with smooth faceting along the NR length. 

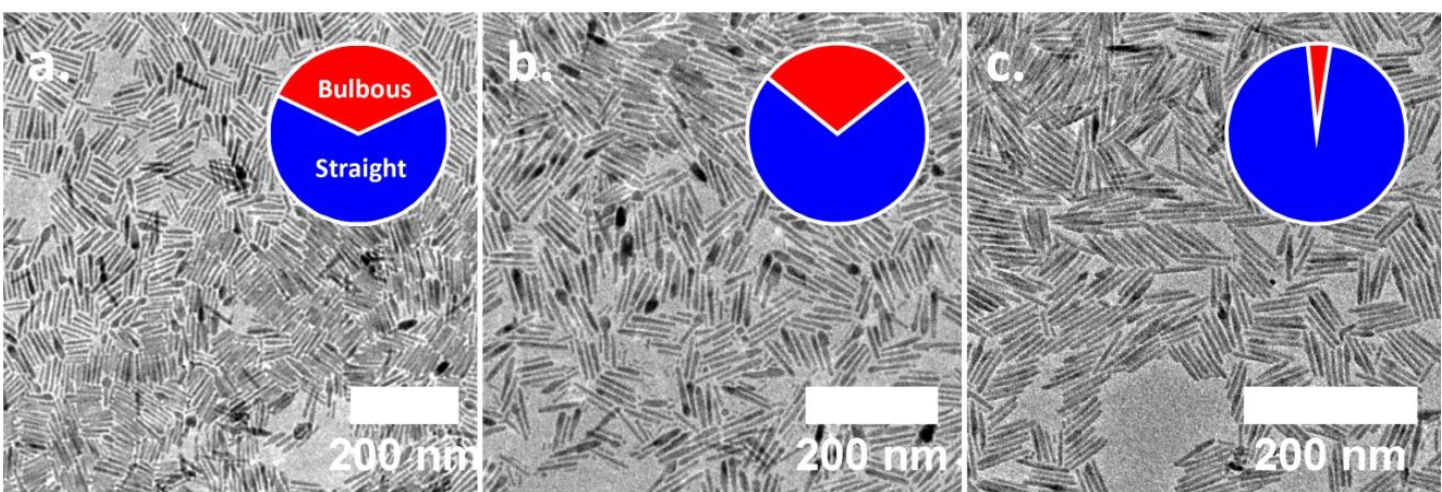

Figure S6. NRs resulting from thermal history experiments. (a) Reaction was allowed to cool to $<200{ }^{\circ} \mathrm{C}$, then reheated to $350{ }^{\circ} \mathrm{C}$ and reacted for 3 minutes. (b) Reaction was heated as normally for $90 \mathrm{~s}$, then cooled to $<200{ }^{\circ} \mathrm{C}$ before reheating to $350{ }^{\circ} \mathrm{C}$ for $90 \mathrm{~s}$. (c) Control reaction in which heat was maintained at set point of $350{ }^{\circ} \mathrm{C}$ for 3 minutes. All reactions were performed with the same seed and precursor concentrations.

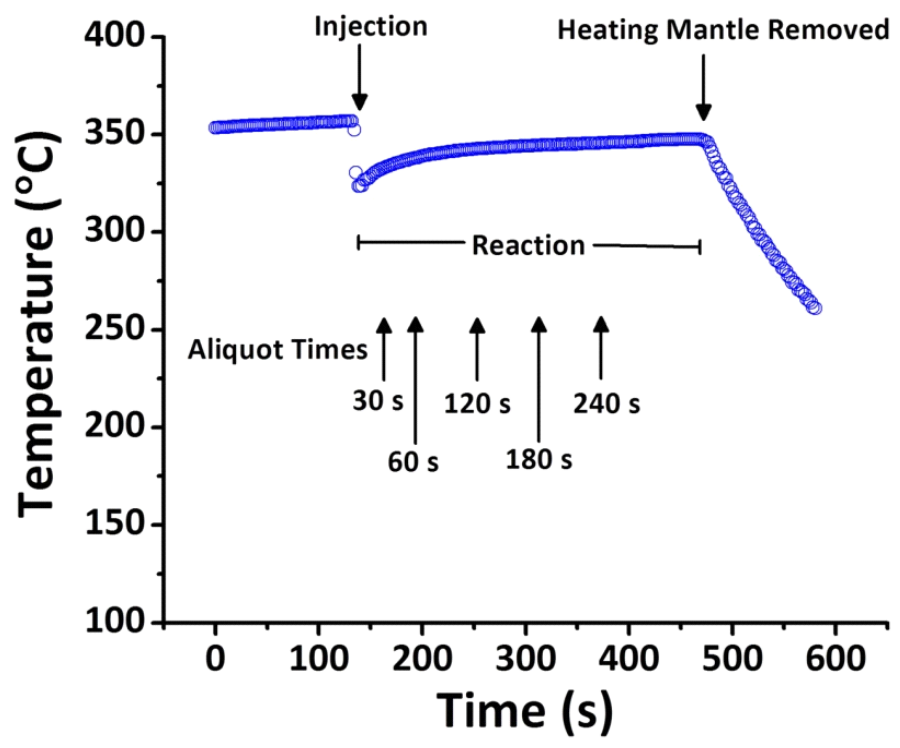

Figure S7. Temperature profile of a typical reaction. The temperature drops substantially upon injection and rises slowly back to the set-point temperature $\left(360{ }^{\circ} \mathrm{C}\right.$ in this case), until the heating source is removed. For reference, the time points used in aliquot experiments are marked on the plot. 

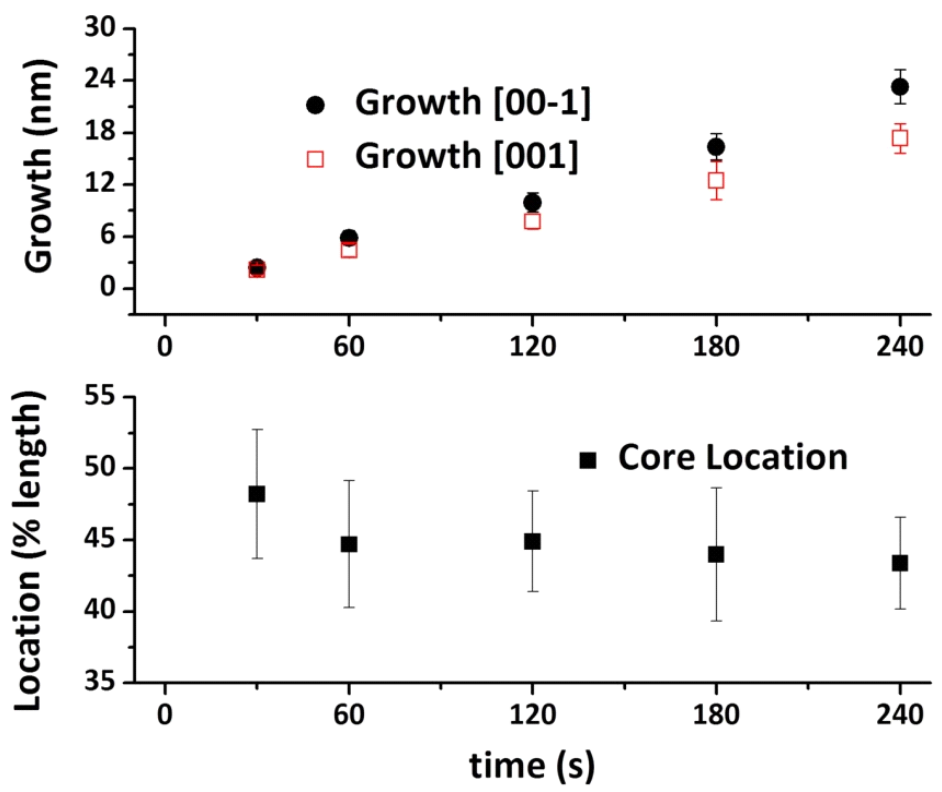

Figure S8. Time-resolved growth dynamics of a CdSe/CdS dot-in-rod reaction. (Top) growth from the core along the (00I ) and (001) are quantified for five aliquots. Slight changes in the reaction rate along these two axes with time (and implicitly temperature, see Figure S7) yield NRs with an increasingly asymmetrically-located core. Error bars represent the standard deviation of the measured values under an assumption of Gaussian distribution.

Significance core "shift". Figure S8 presents data on the growth in the (00I) and (001) directions of CdS and the effect this has on the location of the core with respect to the nanorod length. On the basis of this data, as well as literature reports ${ }^{1}$ and data in Figure 1, it is asserted in the main text that longer reaction times (implicitly higher average reaction temperature, see Figure S7) yield a shift of the core to a position slightly closer to the (001) terminus. Although the probability that this is non-random on the basis of time points at $30 \mathrm{~s}$ and $240 \mathrm{~s}$ in Figure S8 is only 50\%, both data in Figure 1 and intermediate data points in Figure S8 provide further evidence in support of this assertion.

Table S1. Data table of measurements and standard deviations for samples shown in Figure 1. Unless otherwise noted, values are in nanometers.

\begin{tabular}{|l|c|l|l|l|l|l|l|c|}
\hline $\begin{array}{l}\text { Sample } \\
(\#)\end{array}$ & Length & $\begin{array}{l}\text { Core } \\
\text { Length }\end{array}$ & $\begin{array}{l}\text { Core } \\
\text { Width }\end{array}$ & $\begin{array}{l}\text { Core Position } \\
(\% \text { of } \\
\text { Length })\end{array}$ & $\begin{array}{l}\text { Length } \\
(\text { 00I })\end{array}$ & $\begin{array}{l}\text { Length } \\
(001)\end{array}$ & $\begin{array}{l}\text { Length } \\
(100)\end{array}$ & $\begin{array}{l}\text { Length } \\
(10 \mathrm{I})\end{array}$ \\
\hline $\begin{array}{l}\text { Small } \\
(127)\end{array}$ & $14.1 \pm 1.7$ & $2.8 \pm 0.4$ & $2.4 \pm 0.3$ & $51 \pm 5$ & $1.0 \pm 0.2$ & $3.6 \pm 0.34$ & $11.8 \pm 1.6$ & $2.6 \pm 0.5$ \\
\hline $\begin{array}{l}\text { Medium } \\
(147)\end{array}$ & $15.0 \pm 1.2$ & $34.0 \pm 0.4$ & $3.2 \pm 0.3$ & $49 \pm 3$ & $1.1 \pm 0.2$ & $4.4 \pm 0.4$ & $11.8 \pm 1.5$ & $3.3 \pm 0.6$ \\
\hline $\begin{array}{l}\text { Large } \\
(123)\end{array}$ & $25.0 \pm 2.0$ & $6.6 \pm 0.8$ & $5.0 \pm 0.4$ & $45 \pm 5$ & $1.4 \pm 0.6$ & $5.6 \pm 0.4$ & $20.9 \pm 2.0$ & $4.1 \pm 0.6$ \\
\hline $\begin{array}{l}\text { NRs } \\
(114)\end{array}$ & $37.3 \pm 6.8$ & $12.2 \pm 2.0$ & $3.9 \pm 0.4$ & $44 \pm 8$ & $1.2 \pm 0.3$ & $5.8 \pm 0.4$ & $33.3 \pm 6.7$ & $3.6 \pm 1.0$ \\
\hline
\end{tabular}



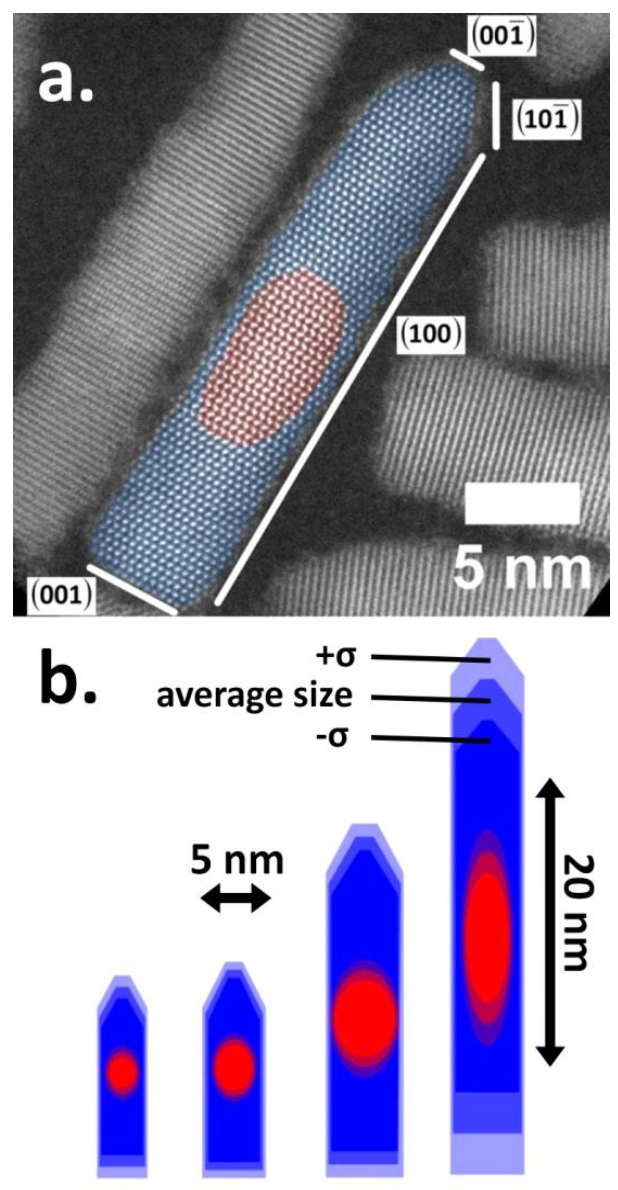

Figure S9. (a) Colorized model of CdSe/CdS dot-in-rod viewed down the [110] zone axis via LAADF-STEM. The dominant surface facets are described in the labeling. (b) Cartoon models of four CdSe/CdS NRs made with different sizes of CdSe seeds. The blue color represents the shell material and red color represents the core. The intermediate shading is the average structure; darker shading is one standard deviation smaller in size for all facets and lighter shading is one standard deviation unit larger in size for all facets. The core position indicated in red also reflects the average position along the length of the NR and the standard deviation in the dispersion of core locations. 


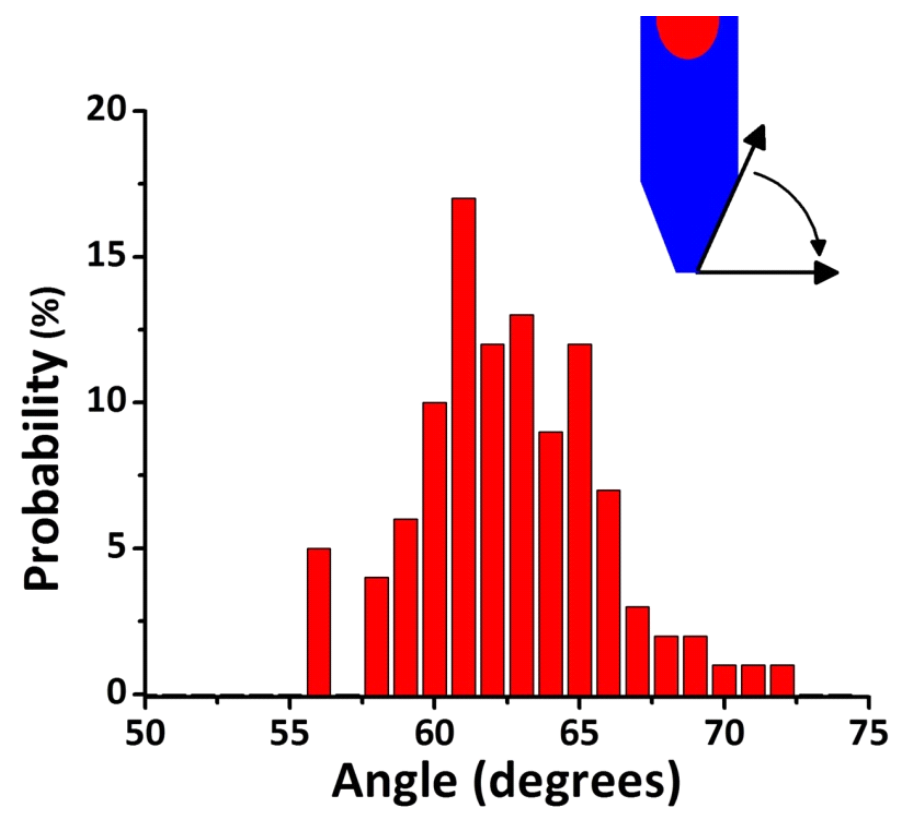

Figure S10. Histogram of measured angles between the (00I ) and (10I ) facets for dot-in-rod sample with $6.6 \mathrm{~nm} \times 5.1 \mathrm{~nm}$ CdSe cores. The ideal angle of a wurtzite CdS crystal at room temperature is $62^{\circ}$. The directly-measured data showed an angle of $62.1 \pm 3.1^{\circ}$. Measurements were performed on samples aligned along the $\langle 110\rangle$ family of zones, but slight changes in the orientation of the nanorod (NR) can generate the dispersion of apparent angles and shown above.

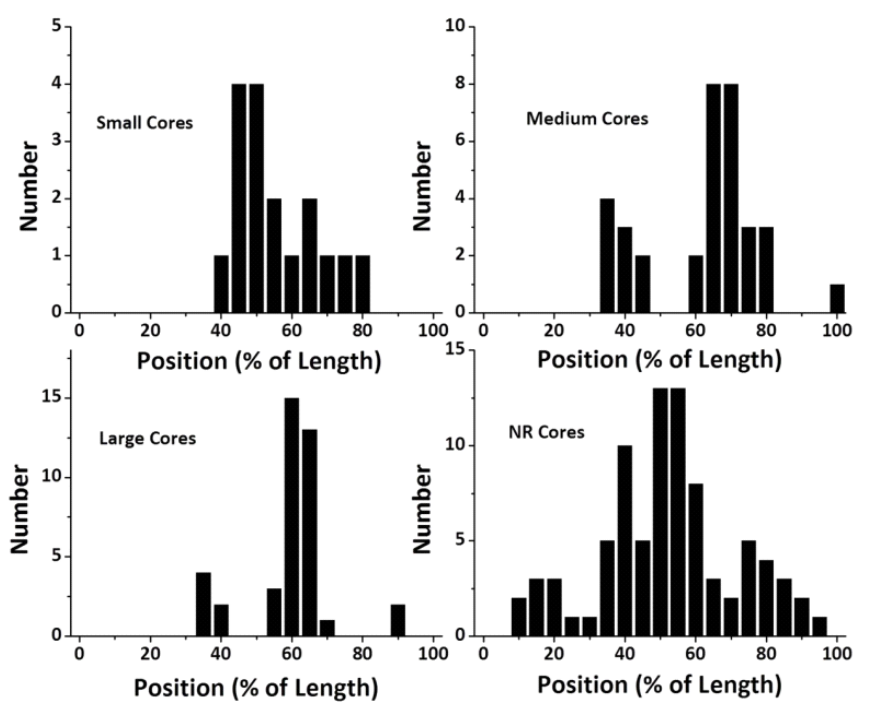

Figure S11. Stacking fault numbers for $2.4 \mathrm{~nm}$ x $2.8 \mathrm{~nm}, 3.1 \mathrm{~nm}$ x $3.9 \mathrm{~nm}, 5.1 \mathrm{~nm}$ x $6.6 \mathrm{~nm}$, and $12.5 \mathrm{~nm}$ x $4.0 \mathrm{~nm}$ (NR) cores of $\mathrm{CdSe} / \mathrm{CdS}$ heterostructures, plotted as a function of the position along the NRs starting from the (001) facet.

Stacking fault analysis. A subset of the stacking faults identified were analyzed to determine their specific structure. The vast majority (>90\%) were intrinsic type 1 and the remainder with intrinsic type 2 . No extrinsic type stacking faults were observed nor were dislocations, presumably because the stacking faults do not terminate inside the NRs. The structure of these stacking fault types is described elsewhere. ${ }^{2}$

Geometric phase analysis. Geometric phase analysis (GPA) was executed in Digital Micrograph using the publically available plugin FRWRTools written by Christoph Koch. ${ }^{3}$ GPA was developed by Hytch et al. and a detailed discussion of 
the technique is available. ${ }^{4}$ The technique was originally developed for phase-contrast transmission electron microscope images but it is suitable for STEM images. Although, scan distortions in STEM images can introduce artifacts into the data, to reduce this, several images from the same NR were acquired using short dwell times and then aligned and summed together using the StackReg plugin within ImageJ. ${ }^{5}$ NRs orientated to the [110] zone axis were analyzed and the same set of Bragg reflections were selected in the Fourier space for all NRs. The reference lattice was defined as a region in the shell (CdS) free from stacking faults. Changes to contrast indicate a distortion of the lattice with respect to the reference region. The two elements of the strain matrix are shown in Figure S10. In each sample a core is visible (red region) depicting the difference in lattice parameter between $\mathrm{CdS}$ and CdSe. The changes in contrast correlates well with the chemical contrast in the ADF-STEM images. 

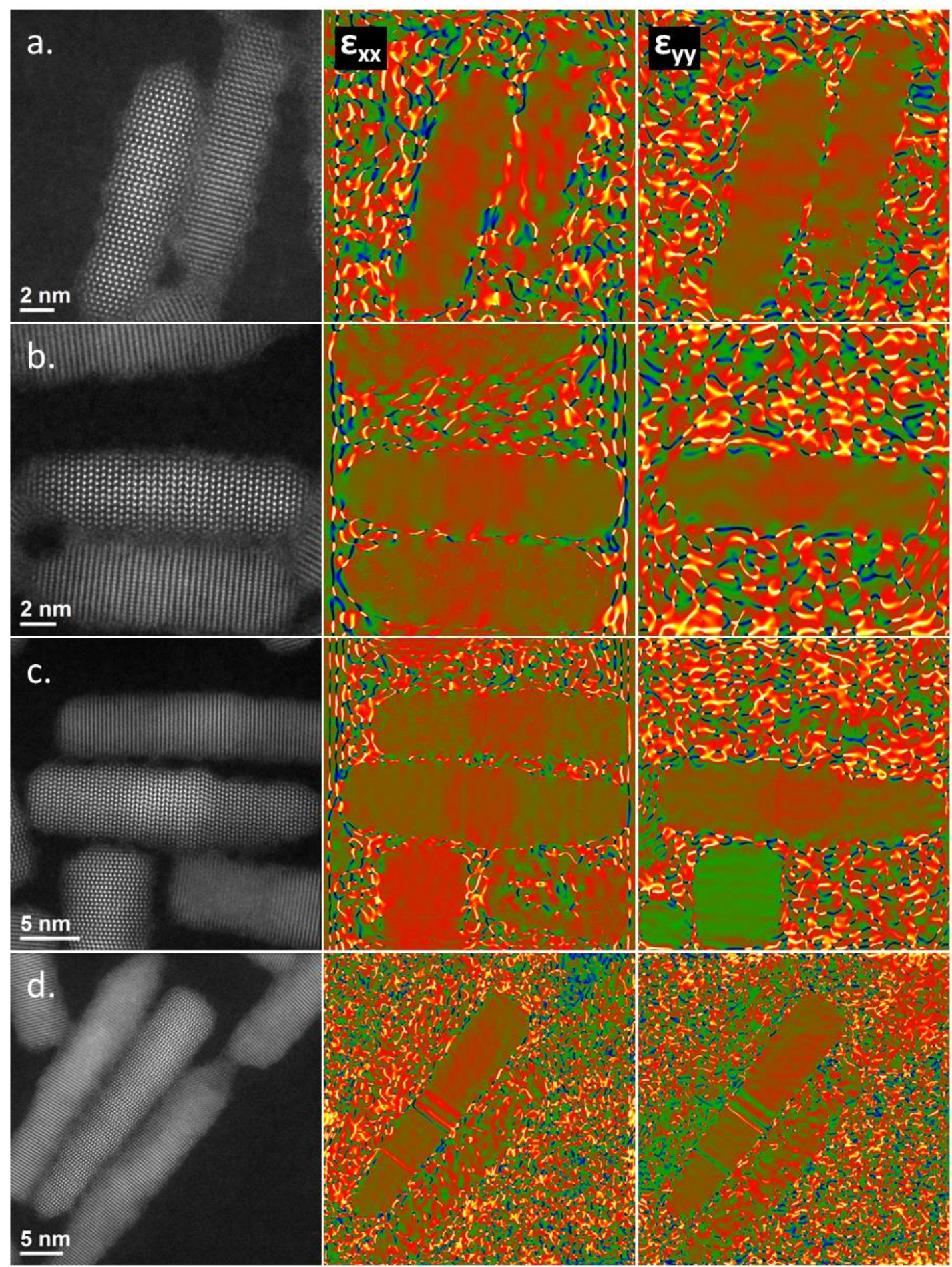

$5 \mathrm{~nm}$

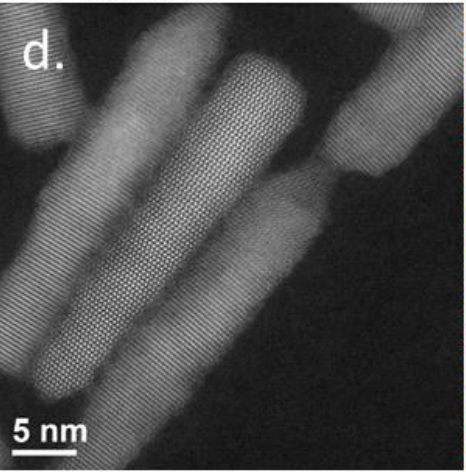

Figure S12. A representative ADF-STEM image from the (a) $2.4 \mathrm{~nm}$ x $2.8 \mathrm{~nm}$, (b) $3.1 \mathrm{~nm} \times 3.9 \mathrm{~nm}$, (c) $5.1 \mathrm{~nm} \times 6.6 \mathrm{~nm}$, and (d) $12.5 \mathrm{~nm}$ x $4.0 \mathrm{~nm}(\mathrm{NR})$ cores core seeded CdSe/CdS heterostructures along with the GPA results depicting elements of the strain matrix. At the CdSe core regions there is an observable change in lattice spacing relative to the CdS shell, which was also demonstrated previously in GPA analysis of $\mathrm{CdSe} / \mathrm{CdS}$ heterostructures. ${ }^{6}$ 
Table S2. Table of strain tensors of CdSe/CdS dot-in-rod and rod-in-rod samples from GPA analysis.

\begin{tabular}{|c|c|c|c|c|c|c|}
\hline Small & & Exx & std. dev. & Eyy & std. dev. & $\mathrm{n}$ \\
\hline & all & 0.043 & 0.009 & 0.046 & 0.014 & 6 \\
\hline & any SF & 0.039 & 0.005 & 0.054 & 0.007 & 4 \\
\hline & no SFs & 0.053 & 0.006 & 0.030 & 0.001 & 2 \\
\hline Medium & & Exx & std. dev. & Eyy & std. dev. & $\mathrm{n}$ \\
\hline & all & 0.044 & 0.009 & 0.047 & 0.007 & 12 \\
\hline & any SF & 0.041 & 0.004 & 0.045 & 0.005 & 8 \\
\hline & no SFs & 0.051 & 0.009 & 0.051 & 0.007 & 4 \\
\hline Large & & Exx & std. dev. & Eyy & std. dev. & n \\
\hline & all & 0.048 & 0.006 & 0.048 & 0.011 & 7 \\
\hline & any SF & 0.048 & 0.006 & 0.048 & 0.011 & 7 \\
\hline & no SFs & NA & NA & NA & NA & 0 \\
\hline Rod & & Exx & std. dev. & Eyy & std. dev. & $n$ \\
\hline & all & 0.046 & 0.022 & 0.053 & 0.014 & 3 \\
\hline & any SF & 0.046 & 0.022 & 0.053 & 0.014 & 3 \\
\hline & no SFs & NA & NA & NA & NA & 0 \\
\hline
\end{tabular}

\section{References}

(1) Talapin, D. V; Nelson, J. H.; Shevchenko, E. V; Aloni, S.; Sadtler, B.; Alivisatos, A. P. Seeded Growth of Highly Luminescent CdSe/CdS Nanoheterostructures with Rod and Tetrapod Morphologies. Nano Lett. 2007, 7, 2951-2959.

(2) Potin, V.; Gil, B.; Charar, S.; Ruterana, P.; Nouet, G. HREM Study of Basal Stacking Faults in GaN Layers Grown Over Sapphire Substrate. Mater. Sci. Eng. B 2001, 82, 114-116.

(3) Koch, C. FRWRTools Plugin http://elim.physik.uni-ulm.de/?page_id=1044 (accessed Jul 9, 2015).

(4) Hÿtch, M. J.; Snoeck, E.; Kilaas, R. Quantitative Measurement of Displacement and Strain Fields from HREM Micrographs. Ultramicroscopy 1998, 74, 131-146.

(5) Thevenaz, P.; Ruttimann, U. E.; Unser, M. A Pyramid Approach to Subpixel Registration Based on Intensity. IEEE Trans. Image Process. 1998, 7, 27-41.

(6) Carbone, L.; Nobile, C.; De Giorgi, M.; Sala, F. Della; Morello, G.; Pompa, P.; Hytch, M.; Snoeck, E.; Fiore, A.; Franchini, I. R.; Nadasan, M.; Silvestre, A. F.; Chiodo, L.; Kudera, S.; Cingolani, R.; Krahne, R.; Manna, L.. Synthesis and Micrometer-Scale Assembly of Colloidal CdSe/CdS Nanorods Prepared by a Seeded Growth Approach. Nano Lett. 2007, 7, 2942-2950. 